\title{
Investigation of Non-Cylindrical Flow in the Region Bounded by Two Coaxial Cylinders of Varying Radii
}

\author{
Vasos Pavlika
}

\begin{abstract}
In this paper non-cylindrical flow variation in swan neck ducts with prescribed flow characteristics will be investigated. The flow will be characterized by the total head, $H(\psi)$ a function of only the stream function, $\psi(x, r)$ and a function of the circumferential velocity $u_{\vartheta}$ where the subscript $\theta$ represents the circumferential component of the velocity vector $\mathrm{u}$ in cylindrical coordinates $(x, r, \theta)$. The cylindrical flow variation is shown to be dependent on the zeros of the Bessel Cross product of order one. It is further shown for a class of swirling flows denoted in this paper as the Stokes-Poisson class that it is theoretically possible for cylindrical flow to be present for any inlet radius $r_{1}$, with the different cylindrical flows given either by circular trigonometric functions or hyperbolic functions and being dependent only on the sign of the separation constant that arises naturally in this analysis.
\end{abstract}

Index Terms-Stokes Stream function, Euler-Poisson Darboux equation, Stokes-Helmholtz class, Stokes-Poisson class, Bessel cross product.

\section{INTRODUCTION}

This is result for which if software and numerical results were to be created and verified this would be lead to a breakthrough into a phenomenon that has never been observed, similar type investigations only being carried out by Taylor [1] but in which a closed form analytical solution has not been given in the literature. The well known equation for the stream function $\psi(x, r)$, of a rotating fluid has been examined with a view of choosing a new dependent variable in such a way that the differential operator becomes the axisymmetric form of the Laplace operator (see for example Arfken [2]), this is advantageous since this makes the setting up of an equivalent integral formula based on Green's first identity, Roach [3], possible since a singular solution to the adjoint equation can be obtained. A suitable choice turns out to be the axial component of the velocity vector $u_{x}$, and for a class of flows including solid body rotation, $u_{x}$, is shown to satisfy the Helmholtz equation subject to an oblique derivative boundary condition. Stoke's stream function $\psi(x, r)$ for steady axisymmetric swirling flow of an incompressible inviscid fluid satisfies the equation:

$$
\frac{\partial^{2} \psi}{\partial x^{2}}+\frac{\partial^{2} \psi}{\partial r^{2}}-\frac{1}{r} \frac{\partial \psi}{\partial r}=r^{2} \frac{d H}{d \psi}-C \frac{d C}{d \psi}
$$

Manuscript received June 21, 2013; revised September 21, 2013.

Vasos Pavlika is with SOAS, University of London, UK (e-mail: vp4@soas.ac.uk). see Pavlika [4], the total Head, $H(\psi)$ (or the total pressure function) and the function $C(\psi)=r u_{\vartheta}$, characterize the nature of the flow, and $\psi$ itself must of course take constant values on any bounding surfaces. Suitable choices for $H(\psi)$ and $(\psi)$ yield a linear equation for $\psi$. The best known of these is:

$$
H=\frac{2 \Omega^{2}}{U} \psi+\frac{1}{2} U^{2}
$$

and

$$
C=2 \Omega \psi / U
$$

which in regions of cylindrical flow, where $\psi$ can be regarded as independent of $x$, can represent a rigid rotation of angular speed $\Omega$, with uniform axial velocity $U$ (see for example Greenspan [5] and Batchelor [6]). This particular case has been the subject of considerable experimental and theoretical work. The experiments of Taylor [1] and Long [7] featured the flow of rotating fluid in a cylindrical tube past a body on the axis. The most general case for which equation (1) is linear is easily seen to be that case with:

$$
\begin{aligned}
& H=a \psi^{2}+b \psi+c \\
& \frac{1}{2} \frac{d C^{2}}{d \psi}=A \psi+B
\end{aligned}
$$

where $a, b, c, A$ and $B$ are constants. Such total pressure and swirl velocity profiles find application in the design of axial flow Turbomachinery (see for example Thwaites [8]) of particular contemporary interest is the flow in the so-called "swan neck" duct connecting the compressor (or turbines) in a multishaft gas turbine. These annular ducts essentially comprise a pair of coaxial cylinders having radii which change monotonically along the axis between constant upstream and downstream values. This gives:

$$
\frac{\partial^{2} \psi}{\partial x^{2}}+\frac{\partial^{2} \psi}{\partial r^{2}}-\frac{1}{r} \frac{\partial \psi}{\partial r}+\left(A-2 a r^{2}\right) \psi=b r^{2}-B
$$

and the corresponding homogeneous equation with $a=0$ (which has implications on $H$ of course) is

$$
\frac{d^{2} F}{d x^{2}}+\frac{d^{2} F}{d r^{2}}-\frac{1}{r} \frac{d F}{d r}+A F=0
$$

where

$$
F=\psi-\left(b r^{2}-B\right) / A
$$

The $H(\psi)$ and $C(\psi)$ profiles in the upstream cylindrical flow 
represent conditions in the exit plane of the final blade row of the upstream compressor. The profiles can be chosen by the compressor designer but are influenced by the feasibility of the blade airfoils necessary to produce them, so whereas early blade rows had values of $H(\psi)$ and $C(\psi)$ independent of $\mathrm{r}$ the so-called "free vortex" blading, most modern machines have some variation across the annulus. The precise nature of these variations is a matter of personal preference, but typically $H(\psi)$ is prescribed as linear function in $r^{2} . \mathrm{H}$ and $\mathrm{C}$ variations based on $\psi$ are equally feasible. The analysis of flow through the swan-neck can therefore be approached using equation (1) and the development described here resulted from attempts to reduce the boundary value problem to the solution of an integral formula involving only boundary values of the speed q. Such reductions hinge on obtaining the so-called fundamental solution to the adjoint equation that is suitably singular. Equation (1) is not particularly suited to this treatment and the following describes a suitable reformulation of the boundary value problem.

In the next section it is shown that the axial component of the velocity vector, $u_{x}$ satisfies the axisymmetric form of the Helmholtz equation, for a class of slightly less general than the $\psi$ linear case, but including the rigid rotation case and sufficiently general to offer worthwhile Turbomachinery application. Boundary values on $u_{x}$ are obtained that are of the oblique derivative type, regular in the Turbomachinery case.

\section{ThE USE OF THE EULER-POISSON-DARbOUX EQUATION IN THE TRANSFORMATION TO THE HELMHOLTZ EQUATION.}

In order for any progress to be made to the development to an equivalent integral reformulation (see for example Pavlika [4] as discussed previously), mention and use of the method of recurrence of Weinstein (1952) [3] on the Euler-Poisson-Darboux equation (EPD) is made. Weinstein solves the singular Cauchy problem for the EPD equation

$$
\nabla^{2} u=\frac{\partial^{2} u}{\partial t^{2}}+\frac{k}{t} \frac{\partial u}{\partial t}
$$

by a technique which included the recurrence formula

$$
u^{(k+2)}(x, t)=\frac{k}{t} \frac{\partial u^{(k)}}{\partial t}(x, t)
$$

where $u^{(k)}$ satisfies equation (4). Consequently if $u^{(k)}$ satisfies equation (4) then $u^{(k+2)}$ will satisfy the equation

$$
\nabla^{2} u^{(k+2)}=\frac{\partial^{2} u^{(k+2)}}{\partial t^{2}}+\frac{(k+2)}{t} \frac{\partial u^{(k+2)}}{\partial t},
$$

letting $k=-1$, gives

$$
\nabla^{2} u^{(1)}=\frac{\partial^{2} u^{(1)}}{\partial t^{2}}+\frac{1}{t} \frac{\partial u^{(1)}}{\partial t},
$$

so with $\nabla^{2}$ identified as $-\frac{\partial^{2}}{\partial x^{2}}, t$ identified as $r$ and $u^{(1)}$ as $F$, then $\frac{\partial^{2} F}{\partial x^{2}}+\frac{\partial^{2} F}{\partial r^{2}}+\frac{1}{r} \frac{\partial F}{\partial r}=0$

which is the Laplace equation in cylindrical polar coordinates. The method of recurrence can be applied to the equation.

$$
\nabla^{2} u=\frac{\partial^{2} u}{\partial t^{2}}+\frac{k}{t} \frac{\partial u}{\partial t}+A u
$$

giving rise to the familiar Helmholtz equation, the dependent variable here, $u$ is of course by equation (5) the term

$$
\frac{1}{r} \frac{\partial F}{\partial r}=\frac{1}{r} \frac{\partial}{\partial r}\left[\psi-\frac{b y^{2}-B}{A}\right]=u_{x}-\frac{2 b}{A}
$$

Formula (5) in its application to equation (4) relies on the precise form of the right hand side of that equation and the absence of functions of $t$ from the left hand side (apart from $u$ itself of course). A direct evaluation shows that:

$$
\frac{\partial^{2} u_{x}}{\partial x^{2}}+\frac{\partial^{2} u_{x}}{\partial r^{2}}+\frac{1}{r} \frac{\partial u_{x}}{\partial r}=\frac{1}{r} \frac{\partial}{\partial r}\left[\frac{\partial^{2} \psi}{\partial x^{2}}+\frac{\partial^{2} \psi}{\partial r^{2}}-\frac{1}{r} \frac{\partial \psi}{\partial r}\right]
$$

and hence

$$
\nabla^{2} u_{x}=\frac{1}{r} \frac{\partial}{\partial r}\left[r^{2} \frac{d H}{d \psi}+\frac{d}{d \psi}\left(\frac{1}{2} C^{2}\right)\right]
$$

which will be linear if

$$
\frac{d H}{d \psi}=b \text { and } \frac{d^{2}}{d \psi^{2}}\left(\frac{1}{2} C^{2}\right)=A
$$

in fact defining $U_{x}=u_{x}-2 b / A$ gives

$$
\nabla^{2} U_{x}+A U_{x}=0
$$

which is the axisymmetric form of the Helmholtz equation. If $A=0$ then

$$
\nabla^{2} U_{x}=2 b
$$

which is the axisymmetric form of the Poisson equation. Flow characterized by equation (6) shall be referred to as the Stoke's-Helmholtz class and flows characterized by equation (7) shall be referred to the Stoke's-Poisson class respectively. The case with $b=0$ leading to the axisymmetric form of the Laplace equation will not be investigated.

\section{BOUNDARY CONDITIONS FOR $U_{X}$}

At any point on a streamline take unit vectors $s$ and $n$, tangential and normal as shown in Fig. 3, (see also Fig. 2 and Fig. 3 for typical annular duct configurations then

$$
\frac{\partial}{\partial n}\left(u_{x}\right)=-\sin \beta \frac{\partial}{\partial x}\left(u_{x}\right)+\cos \beta \frac{\partial}{\partial r}\left(u_{x}\right)
$$




$$
\begin{gathered}
=-\sin \beta \frac{\partial}{\partial x}\left(\frac{1}{r} \frac{\partial \psi}{\partial r}\right)+\cos \beta \frac{\partial}{\partial r}\left(\frac{1}{r} \frac{\partial \psi}{\partial r}\right) \\
=-\frac{1}{r}\left\{\sin \beta \frac{\partial^{2} \psi}{\partial x \partial r}-\cos \beta\left(\frac{\partial^{2} \psi}{\partial r^{2}}-\frac{1}{r} \frac{\partial \psi}{\partial r}\right)\right\} \\
=\frac{1}{r}\left\{\frac{\partial}{\partial s}\left(r u_{r}\right)-\cos \beta\left(\frac{d}{d \psi}\left(\frac{1}{2} C^{2}\right)-r^{2} \frac{d H}{d \psi}\right)\right\} \\
=\frac{\partial}{\partial s}\left(u_{r}\right)+\frac{\sin \beta}{r} u_{r}+\frac{\cos \beta}{r}\left(r^{2} \frac{d H}{d \psi}-\frac{d}{d \psi}\left(\frac{1}{2} C^{2}\right)\right) \\
=\tan \beta \frac{\partial}{\partial s}\left(u_{x}\right)+\frac{u_{x}}{\cos ^{2} \beta} \cdot \frac{\partial \beta}{\partial s}+\frac{\sin \beta \tan \beta}{r} u_{x}+ \\
\frac{\cos \beta}{r}\left(r^{2} \frac{d H}{d \psi}-\frac{d}{d \psi}\left(\frac{1}{2} C^{2}\right)\right)
\end{gathered}
$$

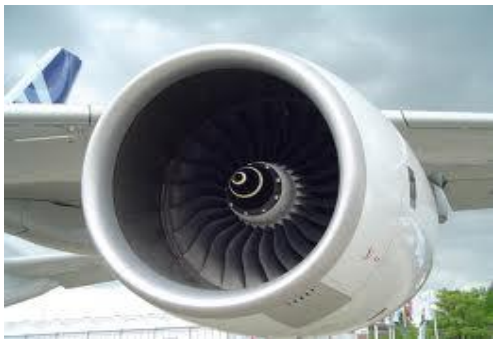

Fig. 1. Typical appication of an annular in aerodynamics.

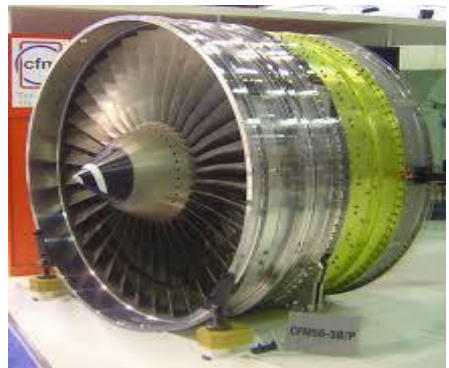

Fig. 2. Typical cross section of an annular duct.

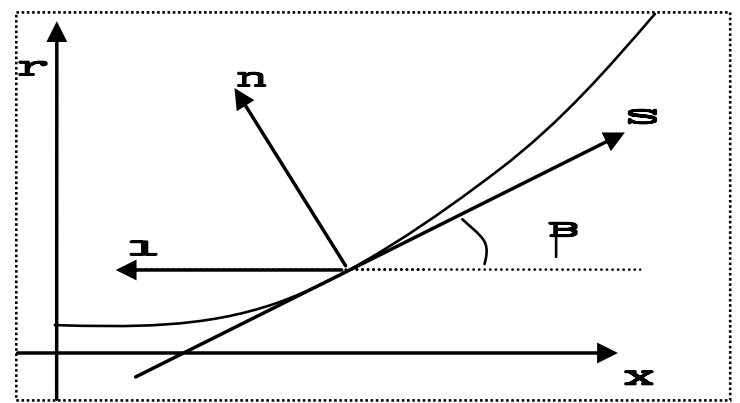

Fig. 3. Showing the tangential and normal vectors to the curve $\psi=$ constant.

If the equation of the streamline is $r^{2}=R(x)$ then this condition can be written as

$$
\begin{gathered}
\frac{\partial}{\partial n}\left(u_{x}\right)=\tan \beta \frac{\partial}{\partial s}\left(u_{x}\right)+ \\
\frac{\cos \beta}{r}\left(\frac{1}{2} u_{x} R^{\prime \prime}(x)+r^{2} \frac{d H}{d \psi}-\frac{d}{d \psi}\left(\frac{1}{2} C^{2}\right)\right)
\end{gathered}
$$

with $\mathbf{I}$ drawn in the direction shown in Fig. 1 the oblique derivative form results:

$$
r \sec ^{2} \beta \frac{\partial}{\partial l}\left(u_{x}\right)=\frac{1}{2} u_{x} R^{\prime \prime}(x)+r^{2} \frac{d H}{d \psi}-\frac{d}{d \psi}\left(\frac{1}{2} C^{2}\right)
$$

For flow of the swan-neck duct type, having known approach conditions, the values of $\psi$ are known on the duct walls, in fact the inner wall can be assigned the value $\psi=0$. Along either boundary wall therefore the boundary condition takes the form

$$
\frac{\partial}{\partial n}\left(u_{x}\right)=\alpha \frac{\partial}{\partial s}\left(u_{x}\right)+\beta u_{x}+\varepsilon
$$

where $\alpha, \beta$ and $\varepsilon$ are known functions and are bounded if $|\beta|$ $<\frac{\pi}{2}$ and $r>0$, which are true of the present application. In considering the upstream and downstream conditions, the possibility of standing waves must be considered. Such waves were exhibited by Taylor [1] and predicted theoretically by Long [7]. Analysis similar to that prescribed by Batchelor [2] shows that in a flow between coaxial cylinders of constant inner and outer radii $r_{1}$ and $\lambda r_{1}$, where $\lambda>0, n$ axial wave components can exist in the duct if $\left(z_{n} / r_{1}\right)^{2}<A<\left(z_{n+1} / r_{1}\right)^{2}$ where $z_{n}$ is the $\mathrm{n}^{\text {th }}$ zero of the Bessel cross product $J_{1}(z) Y_{1}(\lambda z)-J_{1}(\lambda z) Y_{1}(z)$. If such waves are not to exits, A must be chosen less than $\left(z_{1} / r_{1}\right)^{2}$. Sample calculation show that such a restriction does not limit the rotational components normally designed into turbomachinery ducts. Even with non-cylindrical flow, both upstream and downstream of the swan-neck, these conditions can be used to find the distribution of $u_{x}$ on planes normal to the axis placed suitably, relative to the radius variations of the swan-neck itself, and on which $\frac{\partial}{\partial x}\left(u_{x}\right)$ will be expressed as a product of functions in $x$ and $r$. The function in $\mathrm{x}$ may involve circular trigonometric functions and the function in $r$ may involve the Bessel functions $J_{1}(r)$ and $Y_{1}(r)$ or the modified Bessel functions $I_{1}(r)$ and $K_{1}(r)$ all of order one. This is investigated further in the next section.

\section{INVESTIGATING THE NATURE OF NON-CYLINDRICAL FLOW IN THE REGION BOUNDED BY TWO COAXIAL CYLINDERS OF VARYING RADII}

In the following section the possibility of non-cylindrical flow including standing waves being set up in the duct is investigated. The Stokes Stream function $\psi(x, r)$ for steady swirling flow of an incompressible inviscid fluid satisfies equation (1), for the Helmholtz class this becomes

$$
\frac{\partial^{2} \psi}{\partial x^{2}}+\frac{\partial^{2} \psi}{\partial r^{2}}-\frac{1}{r} \frac{\partial \psi}{\partial r}+A \psi=b r^{2}-B
$$

Assuming now that $\psi(x, r)=\psi_{C}(r)+\psi_{N}(x, r)$, where the $C$ subscript denotes Cylindrical flow and the $N$ subscript denotes Non-Cylindrical flow, then

$$
\frac{\partial^{2} \psi_{N}}{\partial x^{2}}+\frac{\partial^{2} \psi_{N}}{\partial r^{2}}-\frac{1}{r} \frac{\partial \psi_{N}}{\partial r}+A \psi_{N}=F(r)
$$

where

$$
F(r)=b r^{2}-B-\left[\frac{d^{2} \psi_{C}}{d r^{2}}-\frac{1}{r} \frac{d \psi_{C}}{d r}+A \psi_{C}\right]
$$


using separation of variables and letting

$$
\psi_{N}(x, r)=r R(r) X(x)
$$

gives:

$$
\frac{1}{X} \frac{d^{2} X}{d x^{2}}+\frac{1}{R} \frac{d^{2} R}{d r^{2}}+\frac{1}{r R} \frac{d R}{d r}-\frac{1}{r^{2}}+A=\frac{F(r)}{r X R}
$$

In order to proceed with the solution it is necessary to put $F(r)=0$ which has implications on $\psi_{C}(r)$ so that:

$$
F(r)=0=b r^{2}-B-\left[\frac{d^{2} \psi_{C}}{d r^{2}}-\frac{1}{r} \frac{d \psi_{C}}{d r}+A \psi_{C}\right]
$$

so that $X(x)$ and $Y(r)$ satisfy:

$$
\frac{1}{R}\left[\frac{d^{2} R}{d r^{2}}+\frac{1}{r} \frac{d R}{d r}\right]-\frac{1}{r^{2}}+A=-\frac{1}{X} \frac{d^{2} X}{d x^{2}}=k^{2}
$$

Since the left hand side is independent of $x$ and the right hand side is independent of $r$ both sides must be equal to a constant. If this constant is chosen positive the $X(x)$ will involve circular trigonometric functions, if chosen negative it will involve hyperbolic functions.

\section{Positive SEParation Constant}

In this case put $k>0$ so that

$$
\frac{d^{2} X}{d x^{2}}=-k^{2} X
$$

and

$$
\frac{d^{2} Y}{d y^{2}}+\frac{1}{r} \frac{d R}{d r}+\left(A-k^{2}-\frac{1}{r^{2}}\right) R=0
$$

which has solutions $J_{1}(\rho)$ and $Y_{1}(\rho)$, where $J_{1}(\rho)$ and $Y_{1}(\rho)$ are the Bessel functions of the first and second kind of order 1 respectively and $\rho=\left(A-k^{2}\right)^{1 / 2} r$. With the general solution $D_{k} J_{1}(\rho)+E_{k} Y_{1}(\rho)$ where the subscript on the constants $D_{k}$ and $E_{k}$ denotes dependence on $\mathrm{k}$. The boundary conditions for $\psi_{N}(x, r)$ are

$$
\psi_{N}\left(x, r_{1}\right)=\psi_{N}\left(x, r_{2}\right)=0
$$

which has implications

$$
\psi_{C}\left(r_{1}\right)=\psi_{1} \text { and } \psi_{C}\left(r_{2}\right)=\psi_{2} .
$$

where $r_{1}$ and $r_{2}$ are the inlet wall radii respectively, then for any value $\mathrm{k}$

$$
D_{k} J_{1}\left(\left(A-k^{2}\right)^{1 / 2} r_{1}\right)+E_{k} Y_{1}\left(\left(A-k^{2}\right)^{1 / 2} r_{1}\right)=0
$$

$$
D_{k} J_{1}\left(\left(A-k^{2}\right)^{1 / 2} r_{2}\right)+E_{k} Y_{1}\left(\left(A-k^{2}\right)^{1 / 2} r_{2}\right)=0
$$

This homogenous system only has the trivial solution unless

$$
\begin{aligned}
& J_{1}\left(\left(A-k^{2}\right)^{1 / 2} r_{1}\right) Y_{1}\left(\left(A-k^{2}\right)^{1 / 2} r_{2}\right) \\
& -J_{1}\left(\left(A-k^{2}\right)^{1 / 2} r_{2}\right) Y_{1}\left(\left(A-k^{2}\right)^{1 / 2} r_{1}\right)=0
\end{aligned}
$$

Given $A, r_{1}$ and $r_{2}$, the question arises "are there any $k$ values which satisfy the condition (12)?" Letting $z=\left(A-k^{2}\right)^{1 / 2} r_{1}$ and $\lambda=r_{2} / r_{1}$, so that (12) becomes

$$
J_{1}(z) Y_{1}(\lambda z)-J_{1}(\lambda z) Y_{1}(z)=0
$$

For $\lambda>1$, the Bessel cross product of order 1 has a monotonically increasing sequence of positive simple real zeros:

$$
z \equiv z_{1}, z_{2}, z_{3}, \ldots ., z_{n}, \ldots, \Rightarrow k_{n}^{2}=A-\left(z_{n} / r_{1}\right)^{2},
$$

where $z_{n}$ is the $n^{\text {th }}$ zero of the cross product, thus

$$
A>\left(z_{n} / r_{1}\right)^{2}>0 \text { or } z_{n}<r_{1} \sqrt{A} \text {, for } k_{n}^{2}>0 \exists
$$

standing waves, thus for given $\mathrm{A}, \mathrm{r}_{1}$ and $\mathrm{r}_{2}, \mathrm{n}$ possible wave components occur where $z_{n}<y_{1} \sqrt{A}<z_{n+1}$.

The $E_{k, n} / D_{k, n}$ ratio follows from

$$
\frac{E_{k, n}}{D_{k, n}}=-\frac{J_{1}\left[\left(A-k_{n}^{2}\right)^{1 / 2} r_{1}\right]}{Y_{1}\left[\left(A-k_{n}^{2}\right)^{1 / 2} r_{1}\right]},
$$

where the double subscript $k, n$ shows dependence on both $k$ and $n$. The $X(x)$ now follows from its ordinary differential equation (9) giving $X(x)=a_{k} \cos \left(k_{n} x+\alpha\right)$, where $a_{k}$ and $\alpha$ are arbitrary constants in this analysis and represent the amplitude of the standing wave and $\alpha$ its phase respectively. The $\psi_{C}(r)$ now follows from its ordinary differential equation (equation (8)) and boundary conditions with unique coefficients for its independent solutions except when

$$
J_{1}\left(\sqrt{A} r_{1}\right) Y_{1}\left(\sqrt{A} r_{2}\right)-Y_{1}\left(\sqrt{A} r_{1}\right) J_{1}\left(\sqrt{A} r_{2}\right)=0 .
$$

This condition for increasing A will apply each time the next wave boundary is reached. At this condition the wave component collapses to a constant and the boundary conditions for the cylindrical flow become singular. In fact $\psi_{C}(r)$ satisfies:

$$
\psi_{C}(r)=\frac{D_{n} y}{\sqrt{A}} J_{1}(\sqrt{A} r)+\frac{E_{n} y}{\sqrt{A}} Y_{1}(\sqrt{A} r)+\frac{b r^{2}-B}{A}
$$

where the $D_{n}$ and $E_{n}$ are constants determined from equation (11). So with $\mathrm{M}$ wave components $\psi_{N}(x, r)$ must satisfy

$$
\begin{gathered}
\psi_{N}(x, r)= \\
r \sum_{n=1}^{M} a_{k, n} \cos \left(k_{n} x+\alpha\right)\left(D_{k, n} J_{1}(\rho)+E_{k, n} Y_{1}(\rho)\right)
\end{gathered}
$$


so that

$$
\begin{aligned}
& U(x, r)= \\
& \frac{1}{r} \sum_{n=1}^{M} a_{k, n} \cos \left(k_{n} x+\alpha\right)\left(D_{k, n} J_{1}(\rho)+E_{k, n} Y_{1}(\rho)\right) \\
& +\frac{1}{r} \sum_{n=1}^{M} a_{k, n} \cos \left(k_{n} x+\alpha\right)\left(D_{k, n}\left(\rho J_{0}(\rho)+J_{1}(\rho)\right)+\right. \\
& E_{k, n}\left(\rho Y_{o}(\rho)+Y_{1}(\rho)\right)-\frac{2 b}{A}
\end{aligned}
$$

\section{Negative SeParation Constant}

In this case the separation constant is put negative so that:

and

$$
\frac{d^{2} X}{d x^{2}}=k^{2} X
$$

$$
r^{2} \frac{d^{2} R}{d r^{2}}+r \frac{d Y}{d r}+\left(\left[A-k^{2}\right] r^{2}-1\right) R=0
$$

in this case letting $\rho=\left(A-k^{2}\right)^{1 / 2} r$ so that the differential equation that $\mathrm{R}$ satisfies is given by:

$$
\rho^{2} \frac{d^{2} R}{d \rho^{2}}+\rho \frac{d R}{d \rho}+\left(\rho^{2}-1\right) R=0
$$

Applying the boundary conditions on $\psi_{N}(x, r)$ implying conditions on the $\psi_{C}(r)$ as shown in the previous section the possibility of non-cylindrical flow upstream and downstream is obtained if and only if:

$$
J_{1}(z) Y_{1}(\lambda z)-J_{1}(\lambda z) Y_{1}(z)=0,
$$

where $\lambda$ and $z$ are as defined previously. Thus a non-cylindrical flow variation exists if $\left(z_{n} / r_{1}\right)^{2}>A>0$ or $z_{n}>r_{1} \sqrt{A}$, so given $\mathrm{A}, r_{1}$ and $r_{2}$, then $\mathrm{n}$ possible non-cylindrical flow components occur, where $z_{n-1}<r_{1} \sqrt{A}<z_{n}$. The $E_{k} / D_{k}$ ratio follows as previous. Solving for $X(x)$ gives

$$
X(x)=F_{k} \cosh \left(k_{n} x\right)+G_{k} \sinh \left(k_{n} x\right),
$$

where $F_{k}$ and $G_{k}$ are constants. So to investigate the possibility of non-cylindrical flow upstream and downstream with the onset of standing waves the separation constant must be taken as positive, if it is taken as negative the noncylindrical flow $\mathrm{x}$ variation will be given in terms of the hyperbolic functions.

\section{ZERo SEPARATION CONSTANT}

In this case $X(x)$ and $R(r)$ satisfy

$$
\frac{1}{R}\left[\frac{d^{2} R}{d r^{2}}+\frac{1}{r} \frac{d R}{d r}\right]-\frac{1}{r^{2}}+A=-\frac{1}{X} \frac{d^{2} X}{d x^{2}}=0
$$

so that $X(x)$ is given by $X(x)=\alpha x+\beta$, where $\alpha$ and $\beta$ are constants. $R(r)$ then satisfies

$$
r^{2} \frac{d^{2} R}{d r^{2}}+r \frac{d R}{d r}-\left(A r^{2}+1\right) R=0
$$

The modified Bessel equation giving rise to the so-called hyperbolic functions as independent solutions $I_{n}(\rho)$ and $K_{n}(\rho)$ (for $\mathrm{n}$ integer), is

$$
\rho^{2} \frac{d^{2} R}{d \rho^{2}}+\rho \frac{d R}{d \rho}-\left(\rho^{2}+n^{2}\right) R=0,
$$

rewriting the differential equation that $\mathrm{R}(\mathrm{r})$ satisfies with $z=$ $A^{1 / 2} r$, gives

$$
z^{2} \frac{d^{2} R}{d z^{2}}+z \frac{d R}{d z}-\left(z^{2}+1\right) R=0,
$$

with $n=1$ giving $I_{1}(z)$ and $K_{1}(z)$ as independent solutions. It appears more convenient to relate this equation to the ordinary Bessel equation of order 1. Introducing the new independent variable $t$ such that $z=-i t$, where $i=\sqrt{-1}$, then

$$
z^{2} \frac{d^{2}}{d z^{2}} \rightarrow t^{2} \frac{d^{2}}{d t^{2}} \text { and } z \frac{d}{d z} \rightarrow t \frac{d}{d t}
$$

and equation (13) becomes

$$
t^{2} \frac{d^{2} R}{d t^{2}}+t \frac{d R}{d t}+\left(t^{2}-1\right) R=0
$$

which is the Bessel equation of order 1 with independent solutions $J_{1}(t)$ and $Y_{1}(t)$ respectively, so the general solution is $R(r)=D_{k} J_{1}(t)+E_{k} Y_{1}(t)$. Performing the same analysis and investigating the Bessel Cross product it is found that non-cylindrical flow is not possible. For the Stokes and Poisson class the same analysis is performed and a remarkable theoretical prediction made.

\section{THE STOKES-POISSON ClASS}

In this case $A=0$ so that $u_{x}$ satisfies $\nabla^{2} u_{x}=2 b$ and $\psi(x, r)$ satisfies:

$$
\frac{\partial^{2} \psi}{\partial x^{2}}+\frac{\partial^{2} \psi}{\partial r^{2}}-\frac{1}{r} \frac{\partial \psi}{\partial r}=b r^{2}-B
$$

once again $\psi(x, r)=\psi_{C}(r)+\psi_{N}(x, r)$, where the $C$ and $N$ subscripts have their previous meanings, using one again separation of variables, it can be shown that

$$
\frac{d^{2}}{d r^{2}}\left(\psi_{C}\right)-\frac{1}{r} \frac{d}{d r}\left(\psi_{C}\right)=b r^{2}-B
$$

and 


$$
\frac{1}{R}\left[\frac{d^{2} \psi_{C}}{d r^{2}}-\frac{1}{r} \frac{d \psi_{C}}{d r}\right]-\frac{1}{r^{2}}=\frac{1}{X} \frac{d^{2} X}{d x^{2}}=k^{2}
$$

Performing the same analysis by applying the boundary conditions it can be shown for a positive separation constant that non-cylindrical flow is not possible, however for a negative separation constant, $X(x)$ and $R(r)$ satisfy:

$$
\frac{d^{2} X}{d x^{2}}-k^{2} X=0
$$

and

$$
r^{2} \frac{d^{2} R}{d r^{2}}+r \frac{d R}{d r}+\left(k^{2} r^{2}-1\right) R=0
$$

giving the general solution as

$$
R_{k}(r)=D_{k} J_{1}(k r)+E_{k} Y_{1}(k r)
$$

and

$$
X_{k}(x)=F_{k} \cosh (k x)+G_{k} \sinh (k x)
$$

Applying the boundary conditions in this case imply that to have a non-cylindrical flow variation given in terms of the hyperbolic functions $\cosh (k x)$ and $\sinh (k x)$ possible

$$
J_{1}(z) Y_{1}(\lambda z)-J_{1}(\lambda z) Y_{1}(z)=0
$$

where $z=k r_{1}$ and $\lambda=r_{2} / r_{1}$. If $z_{n}$ is the $\mathrm{n}^{\text {th }}$ zero of this cross product and using the analysis of section (4) with $z_{n}=k_{n} r_{1}$ the remarkable result that $r_{1}>0$ implies the possibility of a non-cylindrical flow variation. So that for any inlet radius $r_{1}$ there may exist an $\mathrm{x}$ variation in the stream function for the special case which has been referred to as the Stokes-Poisson class. If there is the additional boundary condition that as $x \rightarrow \infty, X(x) \rightarrow 1$, i.e. there is cylindrical flow far upstream, then rewriting the solution to equation (15) as $\alpha e^{k x}+\beta e^{-k x}$ and applying this boundary condition gives $\beta=0$, superposition of solutions gives

$$
\psi_{N}(x, r)=r \sum_{n=1}^{M} A_{k, n} e^{k x}\left(E_{k, n} J_{1}(k r)+F_{k, n} Y_{1}(k r)\right)
$$

where $F_{k, n}$ being a constant and $\psi_{C}(r)$ following from its ordinary differential equation (equation (14)) with boundary condition (11).

\section{CONCLUSIONS}

It has been shown that a cylindrical flow variation may exists in the swan-necked duct by increasing the value of the constant $A$ in the definition of $\frac{1}{2} \frac{d C^{2}}{d \psi}=A \psi+B$ such that different zeros of the Bessel Cross product are encountered.
The cylindrical flow has been shown to be given either by circular trigonometric functions or hyperbolic functions. Even more interestingly it has been shown that for a case of flows characterized and referred to in this paper as the Stokes-Poisson class that a non-cylindrical flow variation will always be present for any inlet radius given by the hyperbolic functions which are of course exponential in nature. All possible signs of the separation constant for both the Stokes-Helmholtz and the Stokes-Poisson classes must be considered as they are each theoretically possible. Furthermore in Pavlika [4] an integral formula based on Green's first identity has been derived to calculate the values of the axial component of velocity from an expression of the form:

$$
4 \pi u_{p}=\iint_{S} u \frac{\partial v}{\partial n}-v \frac{\partial u}{\partial n} d s
$$

where $\Gamma$ is defined as the interior region of the closed surface $\mathrm{S}$ comprising of the duct outer and inner walls and the upstream and downstream planes, $\mathrm{n}$ is the unit outward drawn normal and $\mathrm{v}$ satisfies Helmholtz equation in $\Gamma$ except at $\mathrm{P}$, where it diverges like $1 /\left|\underline{x}-\underline{x}_{p}\right|$.

\section{ACKNOWLEDGMENT}

The author would like to thank his former Ph.D supervisor the late Dr Derek Payne, Director of Oakbark Consultants Ltd and Dr Jane Cousins a former Research Student of Dr Payne for their contribution to the development of this material.

\section{REFERENCES}

[1] G. I. Taylor, "Experiments on the motion of solid bodies in rotating fluids," in Proc. the Royal Society A, vol. 1104, pp. 2213.

[2] G. B. Arfken, Mathematical Methods for Physicists, $5^{\text {th }}$ Edition, Academic Press, 1966.

[3] G. F. Roach, Green's Functions, $2^{\text {nd }}$ Edition, Cambridge University press, 1982, pp. 52-58.

[4] V. Pavlika, "Vector Field Methods and the Hydrodynamic Design of Annular Ducts," Ph.D thesis, University of North London, 1195, chapter: X, pp. 246-260, 1995.

[5] H. P. Greenspan, The Theory of Rotating Fluids, Cambridge University Press, pp. 45-50, 1968.

[6] G. K. Batchelor, An Introduction to Fluid Dynamics, Cambridge University Press, pp. 100-105, 1977.

[7] S. T. Long, "Note on Taylor's "Ink Walls in a rotating fluid," Journal Meteorol, vol. II, pp. 247-249.

[8] B. Thwaites, Incompressible Aerodynamics, chapter: XI, Oxford University Press.

[9] J. B. Diaz, "On Cauchy's problem and fundamental solutions," In Contributions to the theory of partial differential equations, Princeton University Press, pp. 110-115, 1954.

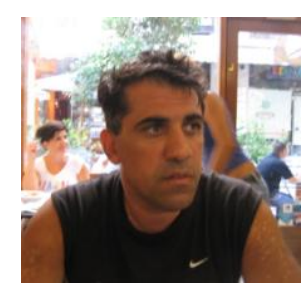

Vasos Pavlika has a B.Sc (Hons) in Mathematics and Physics (1987), an M.Sc in Mathematics (1988), a Ph.D in Applied Mathematics (1995) and a PGCE in teaching Mathematics and Physics in Post-Compulsory Education (1999). He has worked at the University of North London (1988), Newham Sixth Form College (1999), Havering College of Further and Higher Education (2000), The University of East London (2000), The London School of Economics and Political Science (2002-present), The University of Gloucestershire (2002) and The University of Westminster (2003-2010), SOAS, University of London (2003-Present), The University of Oxford (2004-Present), St George's, University of London (2011-2013). Dr Pavlika is a member of the 
WSEAS, an Editor of the Mathematical Journal of the Antarctica and a member of the WCNA. He has a certificate of Merit for a paper presented at the International MultiConference of Engineers and Computer Scientists 2007, Hong-Kong 21-23 March 2007 and Winner of the Best Paper Award for a paper presented at the International MultiConference of Engineers and Computer Scientists 2007, San Francisco 24-26 October 2007 and has over 20 publications including chapters in the following books: The Calculation of Annular Duct Geometries by Prescribing a Velocity Distribution on the Pressure Surface and the Radius of the Suction Surface", pp2375-2383, Lecture Notes in Engineering and Computer Science, Newswood Limited 2007, ISBN 978-988-98671-4-0, Magnetostatic field calculations associated with thick Solenoids in the Presence of Iron using a Power Series expansion and the Complete Elliptic Integrals" Applied Numerical Methods, Springer Verlag 2007 and The calculation of axisymmetric duct geometries for incompressible rotational flow with blockage effects and body forces". Advances in Computational Algorithms and Data Analysis, Springer Verlag 2008. His research Interests include: teaching in HE, theoretical and computational fluid dynamics and Magnetostatics. He is a Fellow of the Institute of Mathematics and its Applications (FIMA, CMath, CSci and CMathTeach), a Fellow of the Institution of Analysts and Programmers (FIAP), a Member of the Institute of Physics (MInstP, CPhys), a Fellow of the British Computer Society (FBCS, CITP and CEng), a Member of the Institution of Electrical Engineers (MIEE and MIET) and a Member of the British Society for the History of Mathematics. 